\title{
Abnormally aggregation-induced emissions observed from hydrogen- and silyl-substituted siloles
}

https://doi.org/10.1515/mgmc-2020-0015

Received December 24, 2019; accepted June 29, 2020.

\begin{abstract}
Silacyclopentadienes (siloles) are currently of great interest because of their intriguing aggregationinduced emission (AIE) characteristics. In this work, abnormally AIE phenomena were observed from silyl- and hydrogen-substituted siloles respectively. We propose silyl-substituent and hydrogen atom effects to explain this via both experiments and theoretical calculations. It was discovered that trimethylsilyl substituents destroyed the $\mathrm{p}$-interactions with the silole core and resulted in the non-fluorescence. Additionally, 1-chloro-1-H-2,3,4,5tetraphenylsilole featuring strong fluorescences in both solid and solution states with high fluorescence quantum yields represents a new potential photoelectric material. This work not only reports the silole with strong fluorescences in both solid and solution states for the first time but also contributs to enrich the AIE research of siloles.
\end{abstract}

Keywords: siloles; aggregation-induced emission; hydrogen effect; silyl-substituents; substituent-fluorescence relationships

\section{Introduction}

Owing to their good biocompatibility, versatile modification strategies, tunable spectral characteristics, and facile processability, organic luminophores are widely

*Corresponding authors: Zhengang Han, Key Laboratory of Bioelectrochemistry and Environmental Analysis of Gansu Province, College of Chemistry and Chemical Engineering, Northwest Normal University, Lanzhou, 730070, P. R. China; e-mail: hanzhengang@nwnu.edu.cn

Kefeng Xie, School of Chemical and Biological Engineering, Lanzhou Jiaotong University, Lanzhou, Gansu 730070, P. R. China;

e-mail:xiekefeng@mail.lzjtu.cn explored and applicated in organic light emitting diodes (OLEDs), fluorescent bioprobes, chemosensors and so on (For recent references on organic luminophores: Guo et al. (2018), He et al., (2018), Lo et al. (2017), Venkatramaiah etal.(2018), Xu et al. (2019), and Yang etal. (2017). However, a phenomenon that is against the practical applications of organic fluorescence has been found by Kasper et al., which is that fluorescence of some organic aromatic compounds in the solution state could be weakened with the concentration of solution increasing, known as aggregation-caused quenching (ACQ) (Birks, 1970; Hong et al., 2011; Thomas III et al., 2007; Yu et al., 2009). Luminophores tend to aggregate in the solution state and then many scientists have to experiment their studies in dilute solutions, which will decrease the sensitivity of bio-sensory and is harmful for practical applications (Jares-Erijman and Jovin, 2003; Mei et al., 2015). Therefore, it is crucial to explore some special materials which can emit strong fluorescence in the solid state.

In 2001, Tang and coworkers observed a fantastic light emission phenomenon from some siloles, such as hexphenylsilole (HPS), which presented strong luminescence in the aggregated or solid state but become weakly emissive or non-emissive in the solution state. This "abnormal" fluorescence behavior is called aggregation-induced emission (AIE) (Luo et al., 2001). It is convinced that these AIE-active siloles possess propeller-like noncoplanar structures and these peripheral "propellers" of siloles can freely rotate in the solution state, which deactivate excited states and then weakens the fluorescence, while this rotated behaviour could be prevented by aggregated molecules and reinforce the fluorescence (Liang et al., 2015; Tang et al., 2001; Zhao et al., 2015). On the other hand, high $\sigma^{\star}-\pi^{\star}$ conjugation interactions between exocyclic carbon atoms and endocyclic silicon atom of siloles result in their low-lying lowest occupied molecular orbitals (LUMOs) (Yamaguchi and Tamao, 1998; Yamaguchi et al., 1998), which is also favorable for their fluorescences in the solid state. 
In this study, considering highly special substituent effect, we synthesized 1-chloro-1-H-2,3,4,5tetraphenylsilole (TPSHC) because the hydrogen atom has the shortest atomic radius and a lower electronegativity, and 2,5-bis(trimethylsilyl)-substituted silole (TPPS) because silyl-substituents exert large steric effects as well as unique electronic effects (Scheme 1) (Fekete et al., 2014; Han et al., 2014, 2018). In previous studies (Gao et al., 2010; Sehuppan et al., 2001), TPSHC was not isolable and often regarded as reactive intermediates so that its photoelectric property was neglected of course. In fact, the structures of two siloles are very similar to that of AIE-active HPS, but show very different light emissions (Figures S7-S10). In detail, TPSHC presents strongly light emissive phenomena in both solid and solution states, while TPSS is non-fluorescent at either solution or solid states. Here we will illustrate the intriguing emissions in a combined experimental and theoretical study.

\section{Results and discussion}

The photographs of three siloles (HPS, TPSS, and TPSHC) are presented in both solid and solution states under natural light and UV irradiation $(\lambda=365 \mathrm{~nm})$, respectively (Figure 1). It can be seen clearly that they show
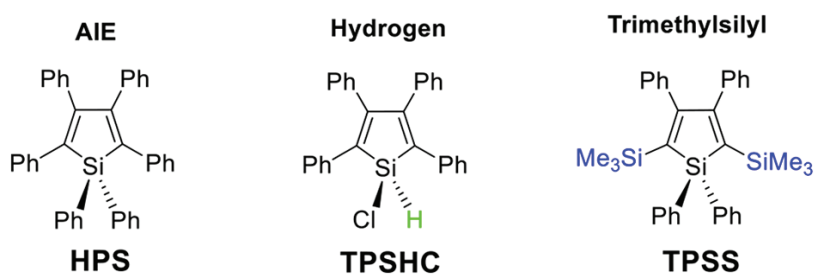

Scheme 1: Chemical structures of HPS, TPSHC, and TPSS.
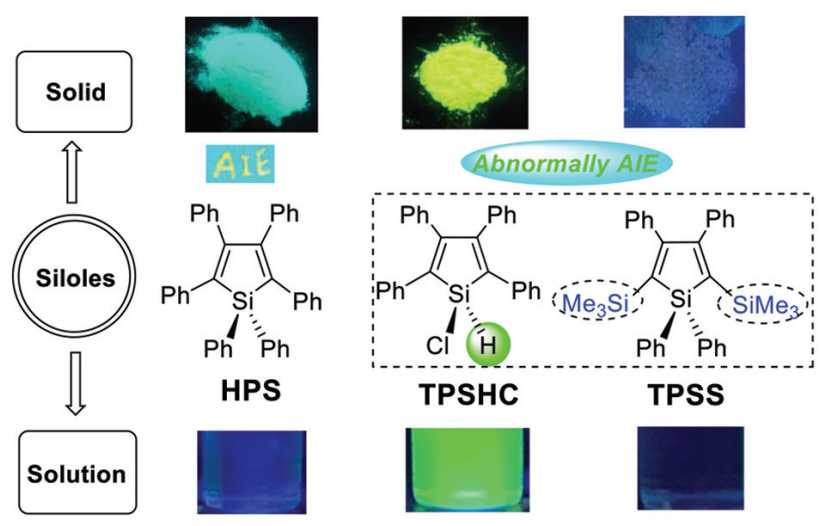

Figure 1: The luminescent spectra of the TPSHC, HPS, and TPPS in the solution state (a) and solid state (b). different light results. Unlike AIE-active HPS, siloles (TPSS and TPSHC) containing inherently special substituents feature light emissions different from previous AIE studies. According to the AIE mechanism, active molecular motions in the solution state serve as a relaxation channel for the excited state to decay non-radiatively, whereas in the aggregated state, the motions could be suppressed greatly by its propeller-like noncoplanar structure (Zhao et al., 2015). At the same time, the conjugated structure is also crucial to the emission for silole, it is demonstrated that substituents at the 2,5-positions determine the emission wavelength to a large degree. So such view cannot be able to perfectly explain the special phenomena. Motivated by this, we investigated the photoluminescence (PL) properties of three siloles (HPS, TPSS, and TPSHC) in both solid and solution states (Figure 2). As well known, siloles are the archetypes of AIE molecules that are strongly fluorescent in the solid state but are either weakly fluorescent or non-fluorescent in solution (Liang et al., 2015; Luo et al., 2001; Tang et al., 2001; Zhao et al., 2015). Although these siloles possess similarly propeller-like noncoplanar structures (Zhao et al., 2015), their PL properties undoubtedly exhibit almost different results. In the solution (THF) state, TPSHC shows a strong emission at $519 \mathrm{~nm}$, HPS shows a very weak fluorescence at $476 \mathrm{~nm}$, while TPSS shows no any fluorescent signals. In the solid state, both TPSHC and HPS are strong fluorescent at 558 and $498 \mathrm{~nm}$, respectively, but TPSS is still not fluorescent. To the best of our knowledge, scarce siloles are brightly fluorescent in both solid and solution states except TPSHC, indicating a new potential material (Figures S8 and S10). The interesting results illustrate the substituent effects in the silole ring play a dominate role on the luminescent properties besides such propeller-like noncoplanar structures. To better understand the particular phenomena, we aim to investigate the structural and electronic characteristics of these siloles by theoretical calculations.

Structures strongly affect the properties of organic materials, including molecular stability, charge carrier mobility, photoluminescence quantum yield, and energy band. Therefore, it is quite indispensible to analysis the structural parameters to illustrate such intriguing light emissive phenomena. The molecular geometry predicted of three siloles by DFT calculations using the Gaussian 03 suite of programs, revision C. 02 at B3LYP/6-31G(d) basis set (Frisch et al., 2004). Some structural factors of HPS, TPSS, and TPSHC are listed in Tables S2-S4, and the optimized chemical structures are shown in Figure 3. The structural parameters of HPS are similar to that reported. After calculations, core silole rings of all 

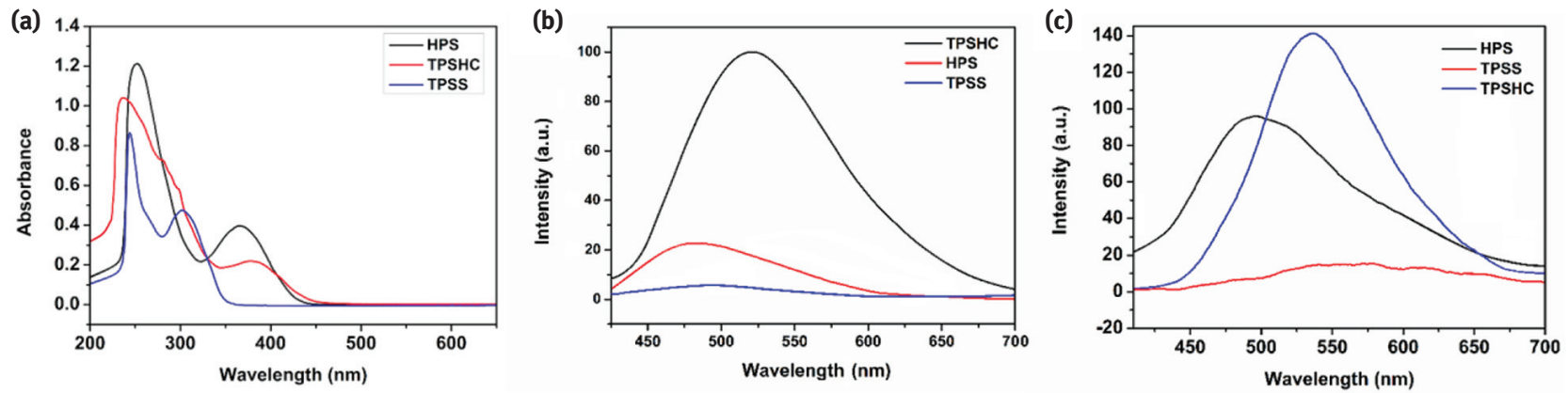

Figure 2: UV-Vis spectra (a) and luminescent spectra of TPSHC, HPS, and TPPS in the solution (b) and solid state (c).

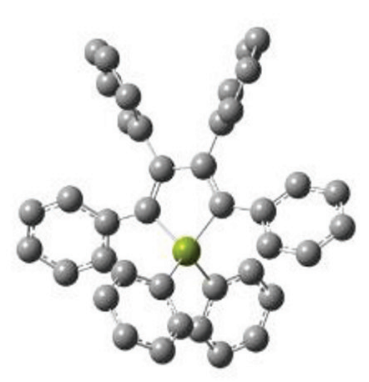

HPS

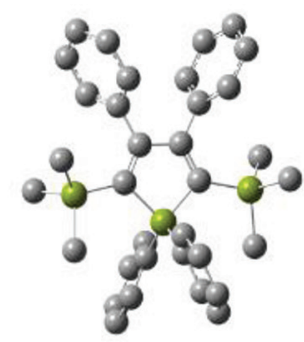

TPSS

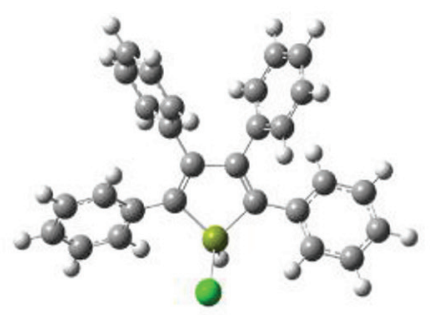

TPSHC

Figure 3: The optimized molecular structures of TPSHC, TPSS, and HPS.

models in TPSS and HPS show great planarity with the dihedral angles no more than $|2.2|^{\circ}$, while the model of TPSHC containing a smaller hydrogen atom presents the poorest planarity (the dihedral angle is $|2.6|^{\circ}$ ) than others. Optimized chemical structures of these three silole models exhibit obviously twisted angle between peripheral phenyl groups and core silole ring. These rotation of phenyl groups which bond to carbons skeleton of silole rings is due to the two-fold symmetrical geometry of core silole ring. The twist degree of phenyl groups at carbon atoms of silole rings is varying, with twist degree from $-87.4^{\circ}$ to $-36.4^{\circ}$ at C2, C3 and C4 positions for all silole models. As for TPSS, the bond lengths of $\mathrm{C}=\mathrm{C}$ double bond (1.359 $\AA$ ) in core silole ring are shorter than that of $\mathrm{C}-\mathrm{C}$ single bond (1.527 ̊), and exocyclic C-C single bond lengths (1.497 ̊̊) are shorter than that of endocyclic $\mathrm{C}-\mathrm{C}$ single bond. This tendency for $\mathrm{C}-\mathrm{C}$ bond lengths has also been observed in HPS and TPHSC models. For C-Si bond length, the exocyclic C-Si bond lengths $(1.908 \AA$ and $1.909 \AA$ at 1,1'-position and 2,5-positions respectively) are shorter than that of endocyclic C-Si bond (1.916 $\AA$ ) in TPSS model. The similar phenomenon is shown in the HPS model, but cannot be observed in the TPHSC model (the Si-C bond length is $1.897 \AA$ ) due to the 1-position substituted by the small hydrogen atom. The average $\mathrm{C}-\mathrm{Si}-\mathrm{C}$ bond angles in core silole ring of TPSS is $92.44^{\circ}$, while these are increased to $93.27^{\circ}$ in TPSHC and decreased to $90.91^{\circ}$ in HPS respectively. As well known, the intramolecular motions and intermolecular $\pi-\pi$ stacking are the main reasons for the differences between AIE and ACQ effects (Caruso et al., 2018). From the above it can be seen that the area of the SiC4 ring for TPSHC is the smallest due to the small hydrogen atom effect, which would lead to a crowded molecular space structure that more effectively restricts their intramolecular motions and suppresses the parallel stacking of aromatic rings obviously in the solid state. This effectively weakens their ACQ effect and enhances their AIE effect to prevent the nonradiative decay. At the same time, compared with the general AIE molecule HPS, the intramolecular motions of TPSHC in the solution state are also restricted intensely because of the cramped and narrow molecular environment, and then contribute enhance the luminescent property as monomers in the solution state. This may be one important reason why TPSHC is more fluorescent than TPSS and HPS in solution.

To increase the photoluminescence efficiency of organic materials in the aggregation state, it is crucial to prevent solid molecules from forming excimers which decrease the self-quenching phenomenon. Results described before demonstrate that all threesilolemolecules present highly twisted nonplanar geometry, especially for the TPSS which shows the largest steric hindrance. 
Theoretically, the intermolecular interaction of AIE-gens could be weakened by these twisted geometries, which could enhance the photoluminescent phenomenon, but we observed that the TPSS shows no photoluminescence in either solution or solid state. In order to clarify this 'abnormal' phenomenon, electronic structures of these three silole molecules are also calculated.

$\mathrm{Yu}$ and coworkers have already analyzed the HPS, 1,1-dimethyl-2,3,4,5-tetraphenylsilole (DMTPS) and 1-methyl-1,2,3,4,5-pentaphenylsilole (MPPS) by both experimental and theoretical methods, they systematically revealed the reasons for AIE luminescence of these siloles (Yu et al., 2005). Some researchers also compared the characteristics of silole and cyclopentadiene, and proposed that the longer maximum absorption wavelength of silole might be due to the higher endocyclic hyperconjugation of silole (Yamaguchi et al., 1996). Additionally, Zhan et al. have already studied some effects caused by different substituents at varying positions of siloles (Zhan et al., 2009), they reported that substitutions at 2,5positions affect optical and redox properties of siloles in a relatively strong level. They proposed that substituents at 1,1-position are related to inductive effects, but show little effect on absorption and emission spectral properties. Tang and coworkers have detailed investigated the silyl-substituent effects on the fluorescence of 2,5-bis (silylethynyl)-3,4-diphenylsiloles (Zhao et al., 2009, 2013) and 2,5-bis(phenylsilyl)-substituted 3,4-diphenylsiloles (Zhou et al., 2013) both in the solution and aggregated state. To describe the difference among HPS, TPSHC and TPSS, some parameters were calculated. The distribution and energy level of molecular orbitals are shown in Figure 3. The highest occupied molecular orbitals (HOMOs) of three models are very closed and mainly distributed on silole ring and 2,5-positions, while the lowest unoccupied molecular orbitals (LUMOs) present some differences. For LUMOs of HPS and TPSHC models, there present an obvious orbital density distribution at $\mathrm{C}-\mathrm{C}$ bond at 2,5-positions of silole ring, while this conjugation is disappeared in the TPSS model. It is noticeable that the introduction of trimethylsilyl groups $\left(-\mathrm{SiMe}_{3}\right)$, which are not the chromophores and destroy the conjugation between 2,5-positons and core silole ring, lead to no fluorescence in any state because 2,5-substituents had the strongest effects on the electronic and optical properties of siloles.

The distribution at $\mathrm{H}-\mathrm{Si}$ bond also be observed at LUMOs of TPSHC model, which might be attributed to the hyperconjugation effect. The relative energy results are also been shown in Figure 4. The highest LUMO $(-1.44 \mathrm{eV})$ and lowest HOMO $(-5.82 \mathrm{eV})$ are shown in TPSS model with the largest energy gap of $4.38 \mathrm{eV}$, while the lowest LUMO $(-2.26 \mathrm{eV})$ is presents in TPSHC model and the highest HOMO $(-5.22 \mathrm{eV})$ is shown in HPS model. The energy gap of TPSHC is the smallest with the data of $3.37 \mathrm{eV}$, which is $0.19 \mathrm{eV}$ smaller than that of HPS. The differences of the energy gaps for these three models could also be observed in the UV-Vis spectrum (as shown in Figure 2a), the redshift of TPSHC and the blue-shift of TPSS compared to HPS. The calculation results demonstrate that the lower LUMO may due to the $\sigma^{\star}-\pi^{\star}$ conjugation between the exocyclic bond and $\mathrm{C}-\mathrm{Si}$ atoms on silole ring and the hyperconjugation effect.
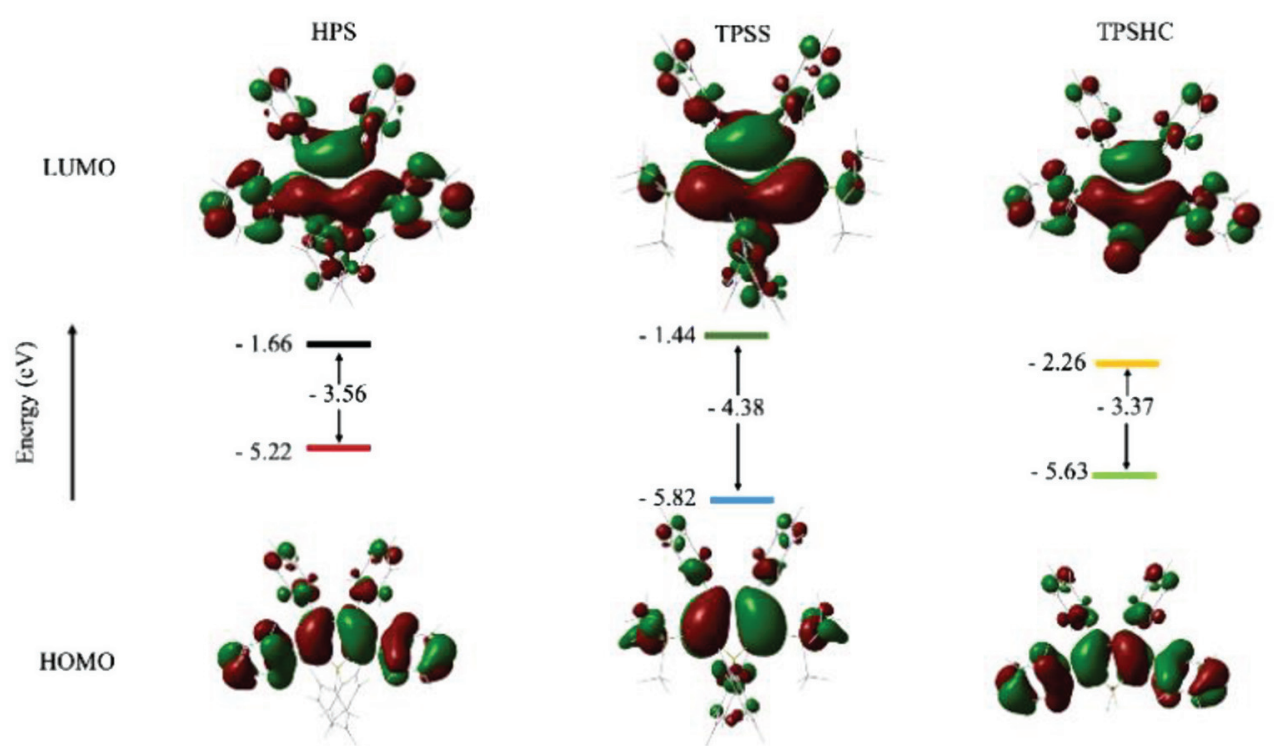

Figure 4: Molecular orbitals distribution of HPS, TPSS, and TPSHC. 
In order to better explain the photoluminescence properties of three siloles in aggregated state, we have also done the calculations in more complex aggregate environment (Figure 5). It can be seen that the intermolecular interactions are in the order as TPSS $>$ HPS $>$ TPSHC.

Nuclear magnetic resonance (NMR) technology has become a powerful tool to illustrate the structural information of organic compounds, especially the silicon nuclear magnetic resonance $\left({ }^{29} \mathrm{Si} \mathrm{NMR}\right)$ spectra can directly exhibit the electronic distribution of the silicon atom (Autschbach et al., 2013; Bonhomme et al., 2012; Rappoport and Apeloig, 1998). As well known, the electronegativity of hydrogen atom (2.20) is very close and slightly larger than that of silicon atom (1.90), so hydrogen atoms often show a little electronegativity in hydrogen-silicon bonds $\left(\mathrm{Si}^{\sigma+}-\mathrm{H}^{\sigma-}\right)$. The ${ }^{29} \mathrm{Si}$ NMR chemical shift for TPSHC (-9.33 ppm) in the silole ring is obviously upfield than in HPS (8.81 ppm) (Figure S4) and TPSS $(7.17 \mathrm{ppm})$ (Figure S6), indicating the increase of electron cloud density after the introduction of hydrogen atom. This is also a very reasonable explanation for the special light emission of TPSHC in solution state.

The above data show that hydrogen atom in TPSHC exhibits a minimum atomic radius and a little electronegativity in hydrogen-silicon bond, which can lead to TPSHC a strong emission because of restricting molecular motions and stabilizing a twisted conformation (Panunzi et al., 2018). On the other hand, hydrogen-silicon bond can increase the $\sigma^{\star}-\pi^{\star}$ conjugation of TPSHC, which can lower the energy gap, and contribute to increase the electronic transmissions. This particular effect in hydrogen-substituted siloles, namely hydrogen effect, can prevent the nonradiative decay and enhanced emission in both solution and solid states.

\section{Conclusion}

In summary, we report the very differently luminescent phenomena for three siloles because of the special substituent effects. Silyl-substituents at the 2,5-positions exert large steric effects and destroy the $p$-interactions between chromophores at the 2,5-positions with the core silole ring, leading to the non-fluorescence of TPSS either in solution or solid states. TPSHC shows novel strong emissions in different states due to the better conjugation from hydrogen atom effects. The finding not only contributes to a more understanding of substituent effects on the aggregation-induced emission of siloles but also provides a useful guidance for designing siloles with better luminescent properties later.

\section{Experimental}

\section{Materials and measurements}

All operations were carried out under an atmosphere of dry argon by using modified Schlenk line and glovebox techniques. All solvents were freshly distilled from $\mathrm{Na}$ and degassed immediately prior to use. The ${ }^{1 \mathrm{H}},{ }^{13} \mathrm{C}$ and ${ }^{29} \mathrm{Si}$ NMR spectroscopic data were recorded on Bruker Mercury Plus $400 \mathrm{MHz}$ NMR spectrometers. Chemical shifts are referenced against external $\mathrm{Me}_{4} \mathrm{Si}\left({ }^{1} \mathrm{H},{ }^{13} \mathrm{C}\right)$ and $\mathrm{SiCl}_{4}\left({ }^{29} \mathrm{Si}\right)$.

\section{Synthesis}

The silole compounds (HPS, TPSHC and TPSS) represent 1,1',2,3,4,5-hexaphenylsilole, 1-chloro-1- $\mathrm{H}$ 2,3,4,5-tetraphenylsilole, and 1,1'3,4-tetraphenyl-2,5bis(trimethylsilyl)silole respectively. Their chemical structures are shown in Scheme 1. The synthetic routes were simplified and improved according to the elegant routes developed by Curtis (1969) and Tomao et al. (1994). All compounds were isolated by recrystallizations instead of previous chromatographic columns in good yields. The detailed procedures can be seen in Supplementary material.

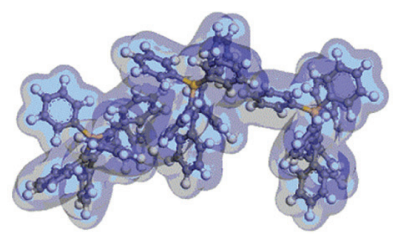

HPS

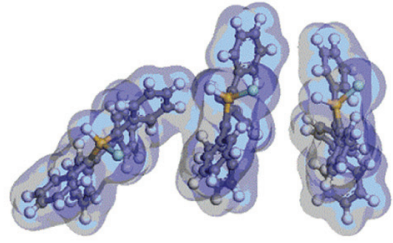

TPSHC

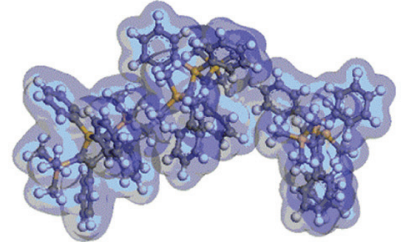

TPSS

Figure 5: Molecular charge density distribution of HPS, TPSS, and TPSHC in more complex aggregate environment. 


\section{Characterization}

All organic compounds were characterized by nuclear magnetic resonance (NMR) and mass spectrum (EI) (see in supporting information page S4, S5 and S6). The NMR spectroscopic data of compounds were determined by Bruke AV 400M NMR spectrometers. The chemical shifts of ${ }^{1} \mathrm{H}$ NMR and ${ }^{29} \mathrm{Si}$ NMR used deuterated $\mathrm{Me}_{4} \mathrm{Si}$ and $\mathrm{SiCl}_{4}$ as references respectively. Mass spectra (EI) were texted on a Shimadzu LCMS-2010EV mass spectrometer. Fluorescence spectra and fluorescence quantum yield were obtained with an Edinburgh FLS920 spectrometer.

\section{Calculation}

All calculations were carried out in the programs of Gaussian 03 (revision E.01). The geometry structures, the optimized 3D chemical structures and molecular orbital plots of three siloles were all optimized by the density functional theory (DFT) method using $6-31+\mathrm{G}(\mathrm{d})$ and UB3LYP basis set.

Acknowledgement: This work was supported by the Natural Science Foundation of China (Grant Nos. 21904106 and 21705117) and Scientific Research Capacity Improvement Project of Northwest Normal University (Grant No. NWNU-LKQN2019-7 and NWNU-LKQN2019-15).

\section{References}

Autschbach J., Relativistic Effects on NMR Parameters, In High Resolution NMR Spectroscopy-Understanding Molecules and Their Electronic Structures. Elsevier B. V., The Netherlands, 2013.

Birks J. B., Photophysics of Aromatic Molecules. Wiley, London, 1970. Bonhomme C., Gervais C., Babonneau F., Coelho C., Pourpoint F., Azais T., et al., First-Principles Calculation of NMR Parameters Using the Gauge Including Projector Augmented Wave Method: A Chemist's Point of View. Chem. Rev., 2012, 112(11), 5733-5779.

Caruso U., Panunzi B., Diana R., Concilio S., Sessa L., Shikler R., et al., AIE/ACQ Effects in Two DR/NIR Emitters: A Structural and DFT Comparative Analysis. Molecules, 2018, 23(8), 1947-1950.

Curtis M.D., Synthesis and Reactions of Some Functionally Substituted Sila- and Germacyclopentadienes. J. Am. Chem. Soc., 1969, 91(22), 6011-6018.

Fekete C.S., Kovacs I., Konczol L., Benko Z., Nyulaszi L., Substituent Effect on the Aromaticity of the Silolide Anion. Struct. Chem., 2014, 25(1), 377-387.
Frisch M.J., Trucks G.W., Schlegel H.B., Scuseria G.E., Robb M.A., Cheeseman J.R., et al., Gaussian 03, Revision E.01. Gaussian, Inc., Wallingford, CT, 2004.

Gao Y., Zhang J., Hu H., Cui C., Base-Stabilized 1-Silacyclopenta-2,4Dienylidenes. Organometallics, 2010, 29(14), 3063-3065.

Guo L., Tian M., Feng R., Zhang G., Zhang R., Li X., et al., InterfaceTargeting Strategy Enables Two-Photon Fluorescent Lipid Droplet Probes for High-Fidelity Imaging of Turbid Tissues and Detecting Fatty Liver. ACS Appl. Mater. Inter., 2018, 10(13), 10706-10717.

Han Z., Li J., Hu H., Zhang J., Cui C., Isolable 1,1-Disubstituted Silole Dianion: a Homogeneous Two-Electron-Transfer Reducing Reagent. Inorg. Chem., 2014, 53(12), 15890-5892.

Han Z., Xie K., Ma X., Lu X., Synthesis and Characterization of a 2, 5-Bis(trimethylsilyl)-Substituted Bis(1,1'-silolide) Dianion. Phosphorus Sulfur, 2018, 193(8), 488-492.

He L., Cao J.-J., Zhang D.-Y., Hao L., Zhang M.-F., Tan C.-P., et al., Lipophilic Phosphorescent Iridium(III) Com plexes as One- and Two-Photon Selective Bioprobes for Lipid Droplets Imaging in Living Cells. Sens. Actuat B-Chem., 2018, 262, 313-325.

Hong Y., Lam J.W.Y., Tang B.Z., Aggregation-Induced Emission. Chem. Soc. Rev., 2011, 40(11), 5361-5388.

Jares-Erijman E.A., Jovin T.M., FRET Imagin. Nat. Biotechnol., 2003, 21(11), 1387-1395.

Liang J., Tang B.Z., Liu B., Specific Light-up Bioprobes Based on AIEgen Conjugate. Chem. Soc. Rev., 2015, 44(10), 2798-2811.

Lo D., Chang C.H., Krucaite G., Volyniuk D., Grazulevicius J.V., Grigalevicius S., Sky-Blue Aggregation-Induced Emission Molecules for Non-Doped Organic Light-Emitting Diodes. J. Mater. Chem. C, 2017, 5(24), 6054-6060.

Luo J., Xie Z.J., Lam J.W.Y., Cheng L., Chen H., Qiu C., et al., Aggregation-Induced Emission of 1-Methyl-1,2,3,4,5-Pentaphenylsilole. Chem. Commun., 2001, 18, 1740-1741.

Mei J., Leung N.L.C., Kwok R.T.K., Lam J.W.Y., Tang B.Z., AggregationInduced Emission: Together We Shine, United We Soar! Chem. Rev., 2015, 115(21), 11718-11940.

Panunzi B., Diana R., Concilio S., Sessa L., Shikler R., Nabha S., et al., Solid-State Highly Effificient DR Mono and Poly-dicyanophenylenevinylene Fluorophores. Molecules, 2018, 23(7), 1505-1517.

Rappoport Z., Apeloig Y. (Eds.), The Chemistry of Organic Silicon Compounds. Wiley, New York, 1998.

Sehuppan J., Herrschaft B., Müller T., The Elusive 7-Silanorbornadien7-ylium: Synthesis and Characterization of Nitrilium and Oxonium Ions Deriving from 2,3-Benzo-7-silanorbornadien-7ylium. Organometallics, 2001, 20(22), 4584-4592.

Tamao K., Yamaguchi S., Shiro M., Oligosiloles: First Synthesis Based on a Novel Endo-Endo Mode Intramolecular Reductive Cyclization of Diethynylsilanes. J. Am. Chem. Soc., 1994, 116, 11715-11722.

Tang B.Z., Zhan X., Yu G., Lee P.P.S., Liu Y., Zhu D., Efficient Blue Emission From Siloles. J. Mater. Chem., 2001, 11(12), 2974-2978.

Thomas III S.W., Joly G.D., Swager T.M., Chemical Sensors Based on Amplifying Fluorescent Conjugated Polymers. Chem. Rev., 2007, 107(4), 1339-1386.

Venkatramaiah N., Kumar G.D., Chandrasekaran Y., Ganduri R., PatilS., Efficient Blue and Yellow Organic Light-Emitting Diodes Enabled 
by Aggregation-Induced Emission. ACS Appl. Mater. Inter., 2018, 10(4), 3838-3847.

Xu H., Zhang H., Liu G., Kong L., Zhu X., Tian X., et al., CoumarinBased Fluorescent Probes for Super-Resolution and Dynamic Tracking of Lipid Droplets. Anal. Chem., 2019, 91(1), 977-982.

Yamaguchi S., Itami Y., Tamao K., Group 14 Metalloles with Thienyl Groups on 2,5-Positions: Effects of Group 14 Elements on Their $\pi$ Electronic Structures. Organometallics, 1998, 17(22), 4910-4916.

Yamaguchi S., Tamao K., Silole-Containing $\pi$-Conjugated Polymers. 5. Theoretical Study of the Electronic Structure of 2,2'-Bisilole in Comparison with 1,1'-Bi-1,3-cyclopentadiene: $\sigma^{\star}-\pi^{\star}$ Conjugation and a Low-Lying LUMO as the Origin of the Unusual Optical Properties of 3,3',4,4'-Tetraphenyl-2,2'-Bisilole. Bull. Chem. Soc. Jpn., 1996, 69(8), 2327-2334.

Yamaguchi S., Tamao K., Silole-Containing $\sigma$ - and $\pi$-Conjugated Compounds. Dalton T., 1998, 22, 3693-3702.

Yang X.L., Xu X.B., Zhou G.J., Recent Advances of the Emitters for High Performance Deep-Blue Organic Light-Emitting Diodes. J. Mater. Chem. C, 2017, 3(5), 913-944.

Yu D., Zhang Z., Wang Z.Y., Ma D., Simple and Efficient Near-Infrared Organic Chromophores for Light-Emitting Diodes with Single
Electroluminescent Emission Above 1000 nm. Adv. Mater., 2009, 21(1), 111-116.

Yu G., Yin S., Li Y., Chen J., Xu X., Sun X., et al., Structures, Electronic states, Photoluminescence, and Carrier Transport Properties of 1,1-Disubstituted 2,3,4,5-Tetraphenylsiloles. J. Am. Chem. Soc., 2005, 127(17), 6335-6346.

Zhan X., Barlow S., Marder S.R., Substituent Effects on the Electronic Structure of Siloles. Chem. Commun., 2009, (15), 1948-1955.

Zhao E., Lam J.-W.-Y., Hong Y., Liu J., Peng Q., Hao J., et al., How Do Substituents Affect Silole Emission? J. Mater. Chem. C, 2013, 1(36), 5661-5668.

Zhao Z., Wang Z., Lu P., Chan C.-Y.-K., Liu D., Lam J.-W.-Y., et al., Structural Modulation of Solid-State Emission of 2,5-Bis (trialkylsilylethynyl)-3,4-Diphenylsiloles. Angew. Chem. Int. Edit., 2009, 48(41), 7608-7611.

Zhao Z., He B., Tang B.Z., Aggregation-Induced Emission of Siloles. Chem. Sci., 2015, 6(10), 5347-5365.

Zhou J., He B., Chen B., Lu P., Sung H.-H.-Y., Williams I.-D., et al., Deep Blue Fluorescent 2,5-Bis(phenylsilyl)-Substituted 3,4Diphenylsiloles: Synthesis, Structure and Aggregation-Induced Emission. Dyes Pigments, 2013, 99(2), 520-525. 